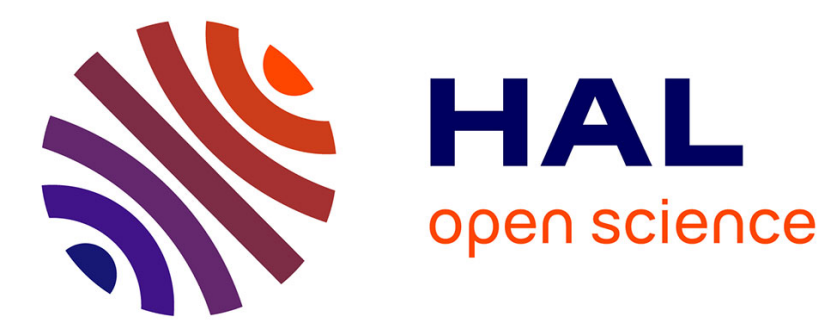

\title{
Innovative interactive flexible docking method for multi-scale reconstruction elucidates dystrophin molecular assembly
}

Anne-Elisabeth Molza, Nicolas Férey, Mirjam Czjzek, Elisabeth Le Rumeur, Jean-François Hubert, Alex Tek, B. Laurent, Marc Baaden, Olivier Delalande

\section{To cite this version:}

Anne-Elisabeth Molza, Nicolas Férey, Mirjam Czjzek, Elisabeth Le Rumeur, Jean-François Hubert, et al.. Innovative interactive flexible docking method for multi-scale reconstruction elucidates dystrophin molecular assembly. Faraday Discussions, 2014, 169, pp.45-62. 10.1039/C3FD00134B . hal-01018071

\section{HAL Id: hal-01018071 \\ https://hal.science/hal-01018071}

Submitted on 3 Jul 2014

HAL is a multi-disciplinary open access archive for the deposit and dissemination of scientific research documents, whether they are published or not. The documents may come from teaching and research institutions in France or abroad, or from public or private research centers.
L'archive ouverte pluridisciplinaire HAL, est destinée au dépôt et à la diffusion de documents scientifiques de niveau recherche, publiés ou non, émanant des établissements d'enseignement et de recherche français ou étrangers, des laboratoires publics ou privés. 


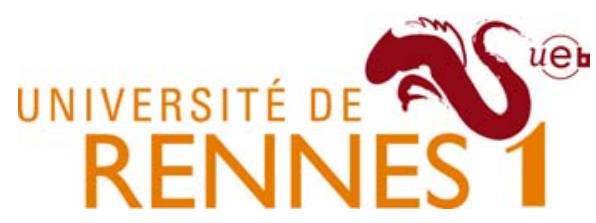

This is the author's final draft post-refeering (post-print)

Find more peer-reviewed articles on our open access repository:

http://hal-univ-rennes1.archives-ouvertes.fr/ 


\title{
Innovative Interactive Flexible Docking method for multi-scale reconstruction elucidates dystrophin molecular assembly
}

Anne-Elisabeth Molza', Nicolas Férey², Mirjam Czjzek ${ }^{3}$, Elisabeth Le Rumeur ${ }^{1}$, Jean-François Hubert ${ }^{1}$, Alex Tek $^{4}$, Benoist Laurent ${ }^{4}$, Marc Baaden ${ }^{4}$, and Olivier Delalande ${ }^{*}, 1$

${ }^{1}$ Institut de Génétique et Développement de Rennes, CNRS, UMR6290, Univ. Rennes 1, Campus Santé, 2 av du Pr Léon Bernard, 35043 Rennes Cedex, France

${ }^{2}$ Laboratoire d'Informatique pour la Mécanique et les Sciences de l'Ingénieur, CNRS, UPR3251, Univ Paris XI, Bât 508, 512 et 502 bis, 91403 Orsay Cedex, France

${ }^{3}$ Laboratoire Végétaux Marins et Biomolécules, CNRS, UMR7139, Univ. Pierre et Marie Curie-Paris 6, Station Biologique, 29682 Roscoff, France

4 Laboratoire de Biochimie Théorique, CNRS, UPR9080, Univ. Paris Diderot, Sorbonne Paris Cité, 13 rue Pierre et Marie Curie, 75005 Paris, France

* corresponding author

\begin{abstract}
At present, our molecular knowledge of dystrophin, the protein encoded by the $D M D$ gene and mutated in myopathy patients, remains limited. To get around the absence of its atomic structure, we have developed an innovative interactive docking method based on the BioSpring software in combination with Small-angle X-ray Scattering (SAXS) data. BioSpring allows interactive handling of biological macromolecules thanks to an augmented Elastic Network Model that combines the spring network with non-bonded terms between atoms or pseudo-atoms. This approach can be used for building molecular assemblies even on a desktop or a laptop computer thanks to code optimizations including parallel computing and GPU programming. By combining atomistic and coarse-grained models, the approach significantly simplifies the set-up of multi-scale scenarios. BioSpring is remarkably efficient for the preparation of numeric simulations or for the design of biomolecular models integrating qualitative experimental data restraints. The combination of this program and SAXS allowed us to propose the first high-resolution models of the filamentous central domain of dystrophin, covering repeats 11 to 17 . Low-resolution interactive docking experiments driven by a potential grid enabled us to propose how dystrophin may associate with F-actin and nNOS. This information gives a peak into medically relevant discoveries to come.
\end{abstract}

\section{Keywords}

Interactive flexible docking, multi-resolution, elastic network model, Small-angle X-ray scattering, dystrophin, actin, neural nitric oxide synthase 


\section{Introduction}

Dystrophin is an essential protein of skeletal muscle (1) encoded by DMD, the largest human gene. Given the cell membrane ruptures observed when dystrophin is lacking (2), as seen in the lethal genetic disease Duchenne Muscular Dystrophy (DMD), dystrophin function is clearly crucial for muscle survival (3). By interacting with cytoskeleton proteins and membrane lipids, dystrophin enables shear stress resistance in muscle cells $(4,5)$. Nevertheless, in the absence of a more exhaustive structural characterization, dystrophin's precise role at the molecular level remains poorly understood. The protein's filamentous state and huge size preclude easy answers, but understanding dystrophin at a molecular level, including its structure, dynamics, and interactions, constitutes a key-step in the optimization of gene therapies for rescuing DMD.

Dystrophin is made up of four domains: a N-terminal actin-binding domain; a central rod domain comprising 24 spectrin-like repeats (R1 to R24) (6); a Cys-rich domain involved in the membrane dystrophin-associated glycoprotein complex; and the Cterminal end. These domains are connected by four hinges. The region of repeats 11 to 17 (R11-17) of the central rod domain is involved in crucial interactions with F-actin, nNOS, and membrane lipids (Fig. 1). It has been previously established that the R11R15 fragment is associated with filamentous actin (F-Actin) $(7,8)$ and that the R16-17 fragment interacts with neural Nitric Oxide Synthase (nNOS) $(9,10)$. Both regions interact with membrane lipids $(11,12)$. However, few structural data are available for the dystrophin protein, and any of these concern the R11-17 region. Interestingly, this region is involved in the hotspot of the in-frame mutations leading to Becker Muscular Dystrophy (BMD) (13). Nevertheless, failure of nNOS recruitment at the membrane is observed in BMD patients, leading to a more severe phenotype (14).

In the present study, we developed a multi-scale reconstruction approach, integrating various experimental and modeling methods. As a proof of concept, the approach was successfully applied, resulting in the structural characterization of the central R11-17 region of dystrophin and its interaction with major protein partners.

With that aim in mind, we first acquired Small Angle X-ray Scattering (SAXS) data from multi-repeat dystrophin fragments. To elucidate the atomic structure of these fragments, we developed theoretical approaches (BioSpring experiments) based on an interactive docking concept (15). This uses an augmented Elastic Network Model (aENM) combining the spring network with non-bonded atoms or pseudo-atoms terms (16). Interactive docking is a user-centred approach that uses advanced 3D interaction devices during a docking simulation to allow application of real-time constraints to the proteins. Complementary to traditional fully-automatic docking approaches, our interactive docking software allows the user to manipulate and drive several flexible proteins to a bound state using his or her own knowledge and expertise of the biomolecular system, but all the while remaining constrained by the underlying biophysical interactions. 
We started by obtaining dystrophin structural data using the aENM coupled to the volumetric data provided by SAXS experiments. Thereafter, the macromolecular complex was constructed using the aENM joined to the electrostatic potential grid computed from experimental structures. Interactive driving of the ligand to the receptor was enhanced using contact data obtained experimentally either from footprint-like mapping (trypsin digestion of lipid-protein complexes) or from in vitro and in vivo cellular experiments on mutant proteins. SAXS-based models provided either ligand (versus F-actin) or receptor (versus PDZ-nNOS) models for dystrophin docking with these two main partners. Beforehand, we performed a rigid low-resolution docking (Attract program) to extensively explore the most probable association modes for the starting structures later considered in the interactive docking step. BioSpring enables both the interactive optimization of the Attract poses following experimental contact mapping, and the evaluation of the effects of introducing flexibility in the molecular models upon their association.

Finally, we present here the first ternary complex composed of F-actin-DystrophinnNOS proteins, revealing structural features that could be related to the dysfunction of the truncated dystrophin forms observed in BMD patients.

\section{Materials and Methods}

Molecular structures. Similarly to our previous structural study (17), homology models for the R11-15 and R16-17 dystrophin fragments were obtained thanks to the I-TASSER web server (18), using an updated template library that includes the structures of the first repeat of both dystrophin and utrophin (19). The molecular dynamics (MD) optimized model of the R15-16 fragment was from our previous work (17). The actin filaments used to perform the docking experiments were 12-actinG polymers built from the CryoEM structures available in the Protein Data Bank (PDB codes 3MFP and 3G37). The nNOS PDZ crystallographic structure (PDB code 1QAU) was completed using homology modeling to fit Lai's experimental sequence (10). This PDZ structure was submitted to Normal Modes Analysis-driven deformations using the elNémo server $(20,21)$ to produce several conformations. All structural data are shown in a schematic cellular context in Figure 1.

Low-resolution rigid docking. Rigid low-resolution Attract docking calculations were performed for both R11-15-F-actin and PDZ-nNOS-R16-17. The most relevant associations between the structures, that could be further associated interactively, were predicted through Attract exhaustive searches. Newly-developed parameters for the Attract force field were used during the docking calculations (22). Placing of the ligands, R11-15 or PDZ-nNOS for each experiment, was achieved thanks to density grids of $25 \AA$ and $10 \AA$. A total of 157 starting points for the dystrophin R11-15 fragment were set all around the actin filament, creating a $140 \AA-$ high cylinder with a $150 \AA$ radius (see Supporting Information). The PDZ-nNOS had 381 starting points around R16-17. For each starting point, the 258 rotation standard value computed for the ligand was used. Final data was a result of 40,506 poses and 98,298 poses respectively for the R11-15-F-actin and PDZ-nNOS-R16-17 docking experiments. Ranking of the best poses was performed by sorting by complex energy and clustering over the ligand position with a root mean square deviation (RMSD) of 
$60 \AA$ (F-actin docking) and $10 \AA$ (PDZ-nNOS). Best theoretical complexes were thus selected for their low energy levels (Attract function) and because they were the most populated clusters. Molecular contacts were defined with a cutoff distance between ligand and receptor of less than $5 \AA$.

Augmented Elastic Network. The coarse graining of initial homology models and experimental PDB structures was performed in accordance to the rules defined by Zacharias (23). The augmented Elastic Network Model (aENM) combines the usual ENM model (harmonic functions) and non-bonding terms (Attract coarse-grain force field) as per our previous work (16) and similar to that proposed by Periole et al. (24) or more recently by Globisch et al. (25). The aENM model supports the use of multiple cut-off distances to designate a final spring network (Fig. 2A). It thus allows the assignation of more or less weight to secondary or ternary structures as needed. In this study, a cut-off distance of $9 \AA$ was used for backbone particles involved in secondary structure elements, and it was reduced to $5 \AA$ for other particles that belong to loops or side chains. The two resulting spring networks were merged into a final aENM model after the particles of both previous aENM models were linked by a spring if they were closer than the cut-off distance of $5 \AA$. All-atom aENM was used for high-resolution reconstruct of the coarse-grain models. Double cut-off ENM had distances of $9 \AA$ for backbone atoms and $3 \AA$ for side chain and loop atoms, and $3 \AA$ was used as the distance cut-off to merge the first two ENM models. Atomic parameters, including charge and radius, were extracted from the AMBER99 force field (26).

Potential grid. Electrostatic potential maps were obtained from APBS calculations (27) achieved for the structure of the receptor molecules (actin or dystrophin R16-17 in each docking experiment) using the Amber force field and $50 \mathrm{mM}$ sodium and chloride ions. APBS-provided electrostatic potential grid data in the OpenDX format are compatible with the BioSpring reading routine.

Experimental volume maps. SAXS scattering curves were analyzed using the ATSAS software suite (28). Twenty molecular shapes were generated using Gasbor's method which is based on Monte-Carlo draws of hard-sphere distributions (one bead per amino acid) paired with cross-validation via scattering curve simulation. Oligomer program was used for weighing the 20 shapes (linear combination solution). Oligomer ranking showed that the best SAXS molecular shapes selected for both R11-15 and R16-17 fragments achieved a high rank (\#1 for both) and high weight percentage (43\% and $31 \%$ contribution to the scattering curve, respectively) as well as a low $\chi^{2}$ value (1.3 and 2.5 , respectively). The volumetric data for the Gasbor molecular shapes were converted into a pseudo-density grid using the MDFF applet of the VMD program (29) at a resolution of $20 \AA$.

Interactive docking. As in our previous studies $(16,30)$, the MDDriver library was used for data exchange between BioSpring calculations and VMD visualizations. The interactive simulations were computed on a 24-core desktop computer using a PHANTOM Omni haptic device (SensAble) for advanced 3D interaction. The 3D aspects of this device make it easier to pick an atom or a coarse-grained particle within the protein, which is intrinsically a 3D object. Haptic features allow the user to 
feel the forces involved in the simulation through force feedback. The BioSpring software manages the aENM model and guides it through a density scalar field (read from data in the OpenDX format) by computing gradients to derive the force to be applied to a particle according to its mass (SAXS grid) or charge (APBS grid). A strict protocol was set up and used for the six experiments done for each scenario (envelope, orientation) to prevent a priori bias that might occur for the interactive fit simulation of models into SAXS volumetric data (for instance placing a specific coiled-coil repeat in a chosen spot of the SAXS envelope). Predefined backbone particle selections of each alpha-helix were used to translate the aENM model into the volumetric grid. The first step of the protocol consisted in the orientation of the aENM filament parallel to the molecular envelope. The entire aENM was then driven into the envelope. The aENM model was then stretched beyond both terminal ends of the molecular envelope, then was let to relax on is own, without interactive intervention, into the volumetric grid and over 20,000 calculation steps. In the case of interactive docking driven by an APBS potential grid, starting positions for the ligand were either set randomly or by using one of the best poses predicted from the preliminary Attract docking calculations. To complete efficient interactive simulations at an all-atom resolution on desktop computers, the OpenCL implementation newly developed by us for the BioSpring software was used.

Structure validation and representation. Validation of SAXS-based coarse-grained models was performed by volume overlap calculations in the Chimera program (31). The final atomic models were evaluated via the SAVeS metaserver (http://services.mbi.ucla.edu/SAVES/), which combine different programs for molecular structure quality control. All figures were realized with VMD or Chimera.

\section{Results}

Interactive fit of homology models into SAXS volumetric data: a quick and efficient method for producing reliable SAXS-based models

An interactive volume fitting procedure (Fig. 2B) enabled us to reconstruct the dystrophin central domain in less than a minute. For the short fragment, obtaining a SAXS-based model was produced even if no interactive driving of the aENM into the volumetric data was performed. The good quality (see Supporting Information) of the models built following the interactive fitting procedure is based on two main features. Firstly, the robustness of the aENM allows us to maintain the secondary structure as well as the particle packing, thus avoiding the collapse of the structure so that reliable solutions could finally be obtained. Indeed, the basic fold of spectrin-like repeats in triple coiled-coil $(17,19)$ was maintained for each dystrophin repeat model. The flexibility of the model still enables individual alpha helices to kink or coiled-coil repeats to rotate towards each other as needed. Secondly, online visualization allows a good evaluation of the volumetric fit between the aENM and the SAXS envelope. This enables the user to discard bad subdomain placement in the experimental volumetric data. It notably made it possible to discriminate between the different $\mathrm{N}$ terminal to C-terminal or C-terminal to $\mathrm{N}$-terminal orientations stemming from the absence of linear shape polarisation of the SAXS volumetric data. The final interactive reconstruction of a SAXS-based protein structure model was done by 
superimposing the all-atom homology model (aENM format) onto the final aENM position obtained by the preliminary coarse-grain interactive fitting, then by calculating the flexible volumetric fit with the all-atom aENM. The final volume overlaps obtained for the R11-15 dystrophin fragment were respectively $60 \%, 86 \%$, and $92 \%$ for the static homology model, flexible coarse-grain model, and flexible allatom model. Overlap percentages for the R16-17 fragment were 84\% (homology), $87 \%$ (coarse-grain aENM), and 91\% (all-atom aENM).

Given the fact that the R15-16 dystrophin fragment is unable to be produced in vitro, thus precluding SAXS data acquisition, we used the previously-obtained MD models (17) to merge the two SAXS-based all-atom models and to propose the first model of the R11-17 fragment of dystrophin. Final SAXS-based atomic models revealed the doglegged form of dystrophin's filamentous structure. It is worth noting that alphahelical structures and coiled-coil arrangements can be maintained despite the sharp kinks between several consecutive repeats (for instance between R14 and R15) that have been observed experimentally (SAXS molecular shapes).

Thanks to the interactive exploration and evaluation of the two possible relative orientations ( $\mathrm{N}$ - to C-terminal end or $\mathrm{C}$ - to $\mathrm{N}$-terminal end) within the SAXS envelopes, we found that the largest kinks are located at the R12-13, R14-15 and R16-17 junctions (linker regions). These linkers are either characterized as straight $\alpha$-helices or as helices bearing a kink of its main axis. Indeed, SAXS volumetric data correspond to a time-averaged molecular shape, and the kinks we observed should correspond to a mean position between two existing extreme conformations. In fact, kinks could have the wrong amplitude but impose the correct relative direction between two consecutive repeats. These flexibility points were considered as "preferential adjustable regions" for the further dystrophin-F-actin docking interactions.

Interactive fit of SAXS-based models into APBS potential grids: how to create a theoretical complex using guidelines produced by systematic low-resolution docking combined with footprint-like experimental data

We explored the association modes between dystrophin and F-actin by analyzing the two available isoforms of filamentous actin when associated with with ADP (3MPF) or with $\mathrm{ADP}, \mathrm{PO}_{4}{ }^{2-}$ and $\mathrm{Mg}^{2+}$ (3G37). We considered these two conformational states to be representative of changes that could occur in vivo in a stretched or relaxed muscle cell, and each was used as static receptor conformations for the interactive docking experiments.

The low-resolution Attract docking experiments provided evidence for the most probable F-actin molecular surfaces that could interact with the R11-15 SAXS-based model. A parallel orientation of both filaments seemed favoured, and the dystrophin fragment is inserted in a F-actin hydrophobic groove and surrounded by small electronegative patches. This latter point is in agreement with the observation that the $\mathrm{R} 11$ to $\mathrm{R} 15$ repeats are among the most positively-charged dystrophin repeats (17). It appears that the three best complexes obtained by Attract, independently from the F-actin structures, ie with or without $\mathrm{Mg}^{2+}$, showed very similar contact 
patterns. The first type of association (clusters ranked \#1 and \#2) showed preferential contacts between dystrophin's R14 and R15 repeats and F-actin, while R11 to R13 showed only poor contacts with the actin filament. The second association mode (clusters ranked \#3) highlights preferential contacts between R11 and $\mathrm{R} 12$ repeats and F-actin. We completed contact frequency mapping by recording the contacts between both proteins with an inter-particle distance $\leq 5 \AA$ in the first 20 molecular complexes clusters obtained by the rigid docking search. We used this mapping to visualize the most probable contact surface on the F-actin. This first docking using Attract did not allow dystrophin flexibility and likely avoids a proper contact between the two filaments, which would explain the discrepancy between the two best solutions with two different regions of dystrophin.

We therefore next used a flexible docking approach with BioSpring. The driving force in interactive docking with BioSpring was provided by an APBS electrostatic potential grid computed on the F-actin experimental isoforms. This docking strategy seems highly relevant because of the predicted impprtance of electrostatics in the interaction of dystrophin with F-actin (12). As an APBS map is calculated for experimental static structures, we considered the F-actin to be a static molecular shape, thus avoiding the miscorrelation in the spatial distribution of volume and electrostatics that would occur if we had proposed a flexible model (aENM) for the receptor filament. Interactive BioSpring docking enabled a flexible adaptation of dystrophin to the static F-actin molecular surface following the identified interaction groove predicted by the Attract-provided static low-resolution search. As shown in Figure 3, graphical rendering of the molecular surface potentials of F-actin during the interactive docking proved to be a powerful help in the interpretation of the dynamic behaviour of the R11-15 dystrophin ligand driven into the potential grid of the F-actin receptor.

The interactive flexible simulation enabled us to obtain a macromolecular complex in agreement with experimental data. In fact, we had previously (33) performed a MSMS analysis after trypsin digestion of R11-15 in interaction with liposomes: the peptide fragments accessible to trypsin proteolysis were predicted to be free of phospholipid interactions and therefore to be potentially involved in another interaction, such as with F-actin. The conclusion then was that helices $B$ and $C$ of repeat $R 13$ and helices $A$ and $B$ of repeat R14 could be involved in an interaction with the actin filament.

We selected the two best association modes proposed by Attract docking as our starting point for the interactive docking simulations. Using these, it appeared that matching the experimental contact restraints would impose a change in the R12 and R13 positioning towards the F-actin filament (Fig. 3). Indeed, in all the Attract poses, a contact between helix $\mathrm{C}$ of $\mathrm{R} 12$ is observed and should be avoided, as the region is expected to preferentially interact with sarcolemma phospholipids. The torsion of the R12-13 linker region allowed us to interactively match the previously-observed experimental contacts, and led to a decrease in the kink angle observed in the SAXS-based model. Looking at the macromolecular complex, it appears that the dystrophin showed a modified topography when compared to the SAXS-based model obtained in solution, as the succession of repeats from R11 to R13 show a rather linear filamentous structure. On the contrary, the kink observed at the R14-15 linker 
region in the SAXS-based model of the R11-15 fragment was maintained in the dystrophin-F-actin complex. Interactive docking led to a continuous contact of both filaments on a dystrophin track defined from R11 helix B to R12 helix A, and from R13 helix B to R14 helix $C$. These results are in agreement with the experimental data collected earlier.

Interactive fit of SAXS-based models into APBS potential grids: how to create a theoretical complex using guidelines obtained from systematic low-resolution docking and combined with mutagenesis experiments

The low-resolution and rigid explorations of the association between nNOS structures and the R16-17 SAXS-based models showed three preferential relative orientations of the PDZ towards the dystrophin fragment (ranked \#1, \#2 and \#6). All three displayed a good correlation with the contact surface identified indirectly on dystrophin by our recent theoretical study (34) and through the mutagenesis experiments performed in vitro and in vivo by Lai and coworkers (10). In comparison to this contact data, the fraction of native contacts in the molecular complexes obtained here are respectively $53 \%, 43 \%$, and $60 \%$. However, even if the PDZ surface in contact with the R16-17 fragment seemed well-defined, the relative orientation of the nNOS PDZ subdomain towards the dystrophin fragment itself remained unfocused, with two of these poses head-to-tail.

The interactive docking experiments associating the nNOS PDZ subdomain to the R16-17 dystrophin fragment (Fig. 4) used the protocol previously detailed for dystrophin/F-actin docking, but with a major change. This time, in addition to dystrophin's flexibility, we also considered the relative flexibility of the receptor (R1617), as both proteins are represented by an aENM. To avoid sliding or rotation of the receptor aENM model towards its APBS potential grid, the terminal-end $\mathrm{C} \alpha$ atoms of the dystrophin fragment were fixed (spatial restraint). The use of such a rigid-flexible heterogeneous aENM model enabled, upon interactive docking, a smooth modulation of the kink characterized experimentally in the SAXS shape at the R16-17 linker region. Long-range driving of the PDZ ligand to the target surface highlighted experimentally is not trivial given the poor contribution of electrostatics expected in the association of the two proteins (34). In these conditions, the three best poses produced by rigid docking were used as starting positions for the PDZ subdomain. Therefore, interactive experiments operate as a precise exploration of the alternative poses, allowing for rapid and accurate convergence on the most probable association mode, completed with online visual checks. The flexible adaptation of both molecular surfaces to each other was observed in each interactive simulation. This interactive flexible approach enabled a better match of the complex initially ranked \#6 by the Attract function score. The fraction of native contacts in this final molecular complex is $80 \%$, corresponding to an increase of $20 \%$ over the corresponding Attract pose. This result underlines the usefulness of flexible modelling in the docking approach.

Upon the interactive exploration of the three best alternative Attract poses, we tried to rank them by perturbation/relaxation trials. Online visualization of the docking simulation confirmed the PDZ pose ranked \#1 as being poorly stabilized despite its 
good Attract score. The pose ranked \#6 by Attract was selected by BioSpring as the most probable lowest energy position of the nNOS subdomain towards the R16-17 dystrophin fragment. Pose \#2 was observed as an intermediate stage between pose \#1 and \#6. Indeed, it could be highly relevant to hypothesise that the two less stabilized poses are transient orientations of the PDZ enabling it to reach its best association mode with dystrophin R16-17.

Interactive fit of high-resolution structures into pseudo-density data: a generic and efficient method to perform all-atom reconstruction of coarse-grain models

All-atom final reconstruction of the full ternary complex was completed either (i) by a fragment-building strategy using a lowest-RMSD superimposition onto the coarsegrained models previously obtained, followed by a short energy minimization step, or by (ii) a volumetric data fit using an all-atom aENM positioned into a grid derived from the coarse-grained models (Fig. 5A). The second approach turned out to be the most efficient for yielding reliable models in the shortest time. This quick coarse-grain to all-atom reconstruction method using BioSpring enabled us to propose structurallystable high-resolution models of dystrophin-F-actin and nNOS-dystrophin complexes obtained from low-resolution interactive docking simulations. The ternary macromolecular complex was obtained by superimposition (lowest RMSD on C $\alpha$ ) of these two atomic models onto the SAXS-based R11-17 dystrophin model (Fig. 5B).

\section{Discussion}

\section{Exploring molecular plasticity: modelling biomolecules under the control of experimental data as if they were clay}

Using the BioSpring software as a powerful model builder enabled us to confirm the robustness of the aENM approach to a user-imposed local deformation of the driving and shaping of biomolecules through interactive simulation. Molecular object inertia or heterogeneous response to the forces applied uniformly to a set of particles can be overcome by pre-selecting the interactive ensemble. An approach consisting of preferential interaction on a selection of protein backbone particles leads to less deformed structures and to a better haptic response, as the particles are more closely connected due to the higher particle-density in their neighbourhood. The models' quality was also enhanced by a multi-cut-off spring-network design which avoids molecular distortion to a very high degree. We also verified that the nonbonding terms (steric and electrostatics) set in the aENM efficiently counterbalance the tendency of ENM to remain in its initial conformation. For instance, the flexibility of the R11-15 model enabled us to induce conformational changes to dystrophin upon its complexation with F-actin.

We should emphasize that molecular packing and folding is not only rendered by the steric term associated to a particle's volume, but they are also structurally encoded by springs in the aENM. Steric clashes, and more seriously structural collapse, are thus easily avoided upon molecular docking or volumetric data fitting when compared to what can be observed with other fitting methods such as MDFF (35). BioSpring interactive docking into volumetric data enables the calculation of either intra- 
molecular or inter-molecular interactions using non-bonding terms of the aENM representation, in contrast to a simple volume match guided by a density map. This considerably improves the quality of the flexible fit under experimental volumetric restraints, notably aiding in the judicious placing of loops in biomolecular models. We tested BioSpring experiments as an efficient and quick generic method for multi-scale reconstruction by generalizing the approach, combining flexible molecular models and a spatial distribution fitting frame. The speed and the reliability of the models fit convergence into the volumetric target also make an offline procedure possible. This is comparable to the recently-published Internal Coordinates Flexible Fitting (ICFF) method (36), which is several orders of magnitude faster than a Molecular Dynamics approach such as MDFF. However, detailed information about flexible regions is required before using the ICFF method to its full efficiency. Our more generic aENM approach allows limited flexibility in amplitude but does not necessarily impose the set of a pre-defined flexible ensemble, since the regions bearing an increased flexibility are intrinsically related to regions with lower density of particles. BioSpring is still also much quicker than MDFF thanks to coarse-graining and code optimization (OpenCL).

A possible limitation in the long-range driving in interactive docking experiments could arise either from biomolecular system size and complexity or from a lack of importance of electrostatics in the association of the two studied partners. By displaying experimental contacts on the ligand and/or the receptor, the interactive docking procedure was revealed to be more rapid and more accurate than without them. The interactive procedure enabled us also to drive the association by proposing complex moves that are difficult or impossible to set up with traditional or offline docking approaches. For instance, during dystrophin-actin docking, the rotation of a part of the filament around its main axis (R12-13) was necessary to achieve a reliable match with data proposed by experimental contact mapping. Moreover, distancing a ligand subdomain from an unwanted position in the proximity of the receptor, as performed for the R15 repeat towards F-actin, is not easy to achieve in traditional docking programs, usually because they intrinsically force the matching of both the ligand and the receptor.

It is logical to note that the correlation between the experimental data increased if the flexibility was also extended from the ligand to the receptor contact surface, simply keeping its backbone fixed. The modelling of biomolecular matter can be easily set up by the design of a rigid/flexible heterogeneous construction from the BioSpring software commands, which distinguish between static and dynamic particle sets. This BioSpring option enabled us to considerably speed up the interactive calculation rate for very large systems, with the simulation being faster in proportion to a smaller size of the dynamic particle set.

Exploring the dystrophin's interactome: modelling the protein complex formed in the region of a hotspot of a genetic pathology.

In the final ternary complex, three major observations brought crucial understanding to the macromolecular system involving dystrophin. The first concerns the weak contacts of the R15 repeat with F-actin and of the R16 repeat with nNOS. Those two 
repeats seem to be important for structuring the dystrophin filament, but also emphasize a structural frontier between the contact positions of each protein partner. This may be related to the atypical feature of the R15-16 linker region, a loop twelveresidues long which is probably at the origin of its in vitro instability. The second observation highlighted by our macromolecular model deals with the relative positioning of dystrophin and actin filaments with respect to each other. While the Nterminal end of dystrophin R11-15 fragment is laid out parallel to the F- actin, mainly interacting with only one of the two actin filaments, the C-terminal end kinks at the linker regions of the final repeats, disengaging from F-actin. This contact pattern extends along a distance of $150 \AA$ and with a coiling of both dystrophin and actin filaments over an angle of $180^{\circ}$. This observation is compatible with both of the Factin isoforms considered in our study, and indicates that dystrophin does not seem to bridge the two dimerizing filaments of $\mathrm{F}$-actin. The third main observation concerns the relative positioning of F-actin towards the nNOS PDZ subdomain. In the final macromolecular complex, the dystrophin R11-17 contact surfaces interacting with Factin and nNOS are oriented in a similar manner. This could emphasize the structuring of another interaction subdomain, that of the potential regions in contact with phospholipids. In the truncated BMD forms of dystrophin, this polarised 3D organisation could be affected, resulting in the loss of efficiency in dystrophin's essential role as a scaffold protein for the muscle cell membrane.

\section{Conclusion}

The interactive flexible method based on the BioSpring software and presented here allows one to easily swap from a one-residue coarse-grain level (SAXS molecular shap in the Gasbor method) to the Attract coarse-grain level, finally coming up with a high-resolution molecular description. The approach is a highly reliable multiresolution model builder for the construction of large molecular assemblies. In the future, the interactive simulations of the fitting of molecular models to SAXS data could be greatly improved by an instant display of the scattering curve calculated for a given state of the aENM.

We present here the first models of dystrophin fragments in solution, based on SAXS measurements. We also associate, using an interactive flexible docking approach, the dystrophin R11-15 SAXS model with the F-actin atomic structure, obtaining a final macromolecular complex that correlates well with experimental contact mapping. We also propose the first molecular model for the association of dystrophin R16-17 to the nNOS PDZ subdomain, which is well supported by in vitro and in vivo data. The ternary complex involving the BMD hotspot region of dystrophin (R11-17) with two of its main protein partners was modelled by combining theoretical and experimental information, leading to a highly-reliable structural model.

\section{Availability}

The BioSpring software is an open source program available online at http://sourceforge.net/projects/biospring.

\section{Supporting information}


Figure representing Attract docking starting points and table for the SAVES structural analysis are accessible as supplementary data.

\section{Acknowledgements}

The authors are indebted to beamline staff for precious help on the different SAXS beamlines : Adam Round and Petra Pernod at ID14-EH3, ESRF, Grenoble, France and Javier Perez and Pierre Roblin at SWING, Soleil, St Aubain, France.

\section{Funding}

The Association Française contre les Myopathies (AFM) and Rennes Métropole provided financial support, Anne-Elisabeth Molza PhD fellow is funded by an AFM grant. Computer time was provided by GENCI (IDRIS and CINES, grant DYSIM 076816).

\section{Figures}

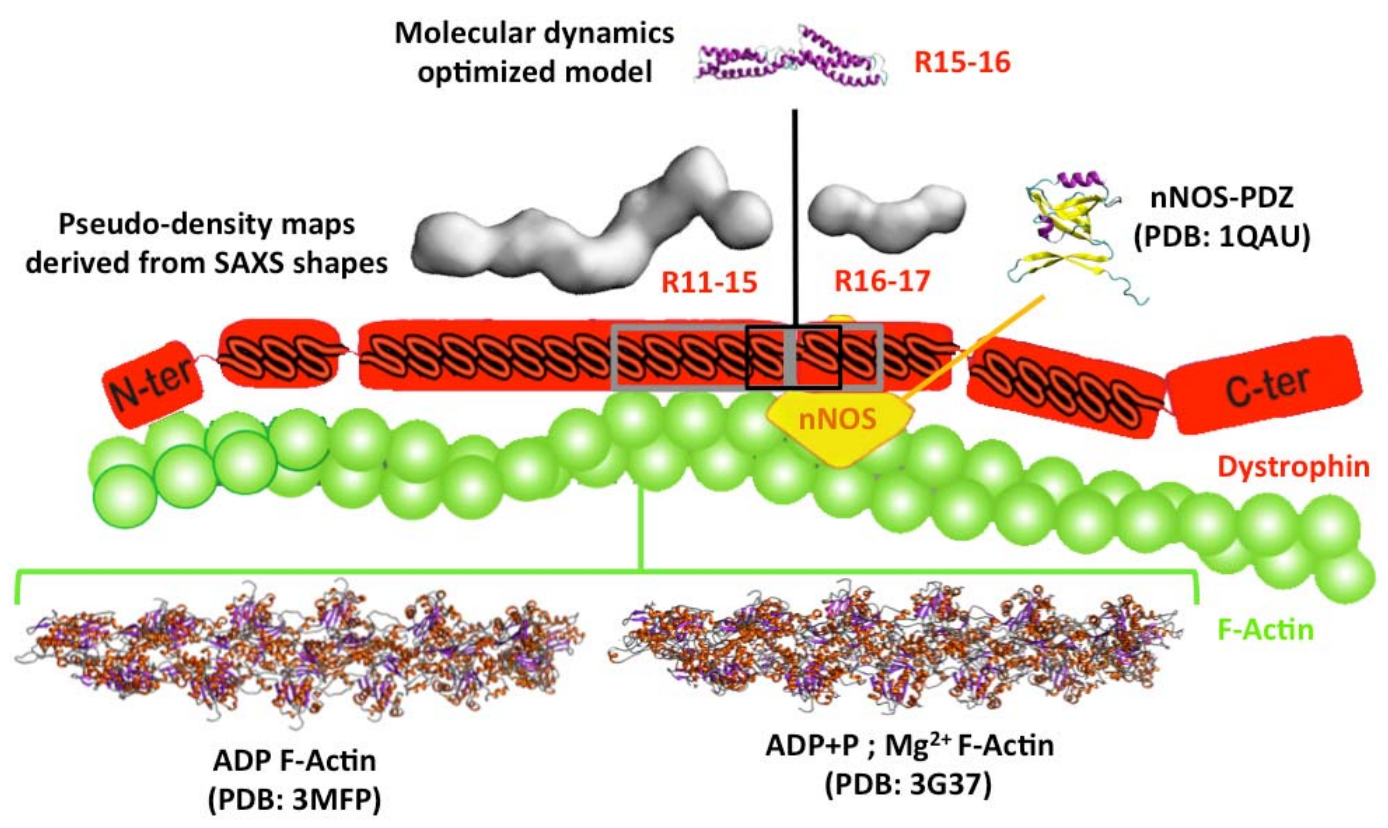

Figure 1: The interaction between dystrophin, nNOS and F-Actin. Structural information for the nNOS-PDZ domain and F-Actin filaments are respectively provided by X-ray diffraction and CryoEM experiments. PDB codes are 1QAU for nNOS and 3MFP or $3 G 37$ for F-Actin. Dystrophin structural data were obtained in solution from Small-angle X-ray Scattering (SAXS) or in silico from homology modelling followed by molecular dynamics optimization. 
A

Homology Model

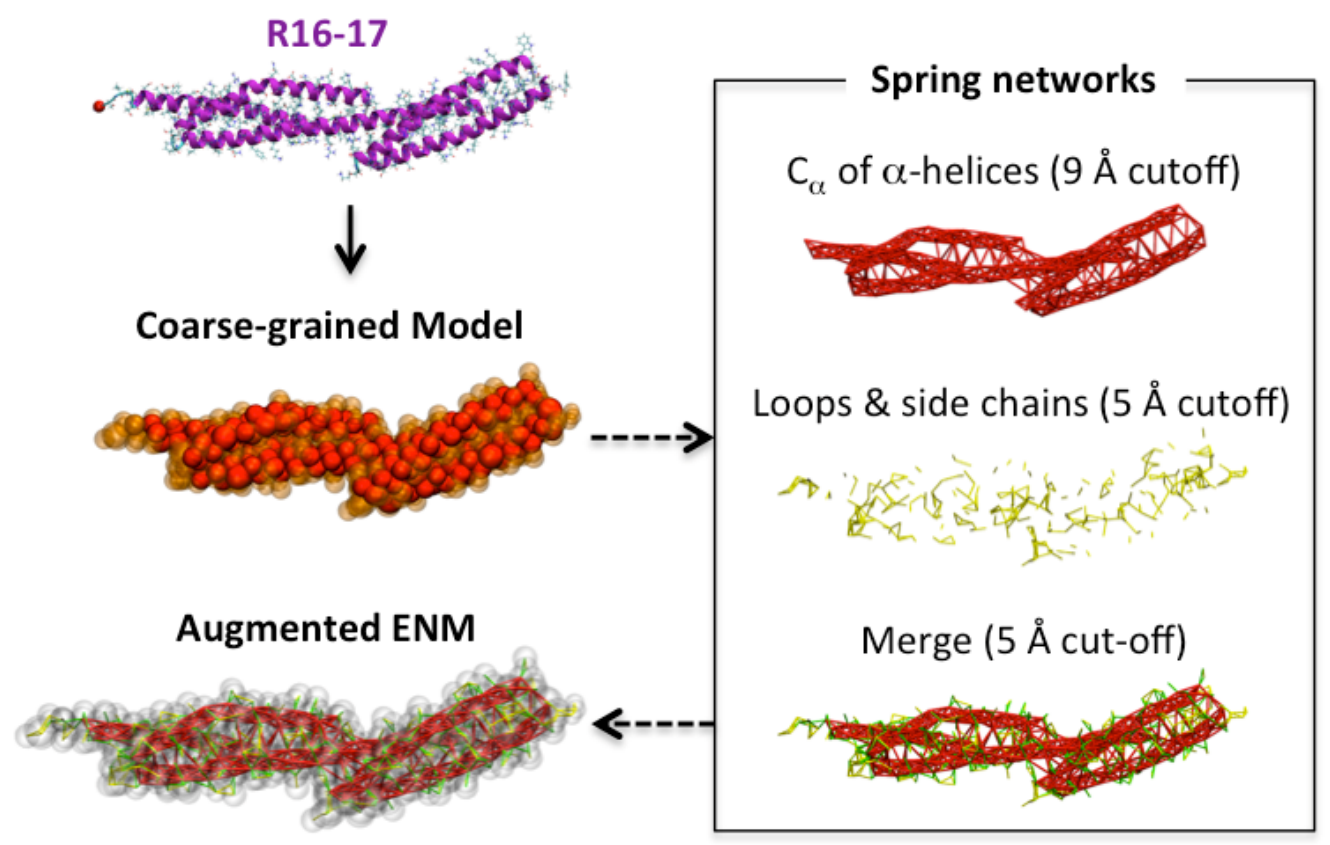

Figure 2A: Protocol for building a coarse-grained augmented Elastic Network Model (aENM) of the R16-17 repeat fragment of dystrophin. From the all-atom homology model provided by the i-TASSER server, a low-resolution model was obtained using the Attract force field parameters developed by Zacharias et al (23). A multi-cut-off spring network can be designed to discriminate between the most flexible and the most rigid regions. Here we chose to stabilize secondary ( $\alpha$-helices) and ternary (coiled-coil) structures. The completion of an aENM model is possible through combining the steric and charge parameters assigned to the pseudo-atoms with the springs defining the ENM. 

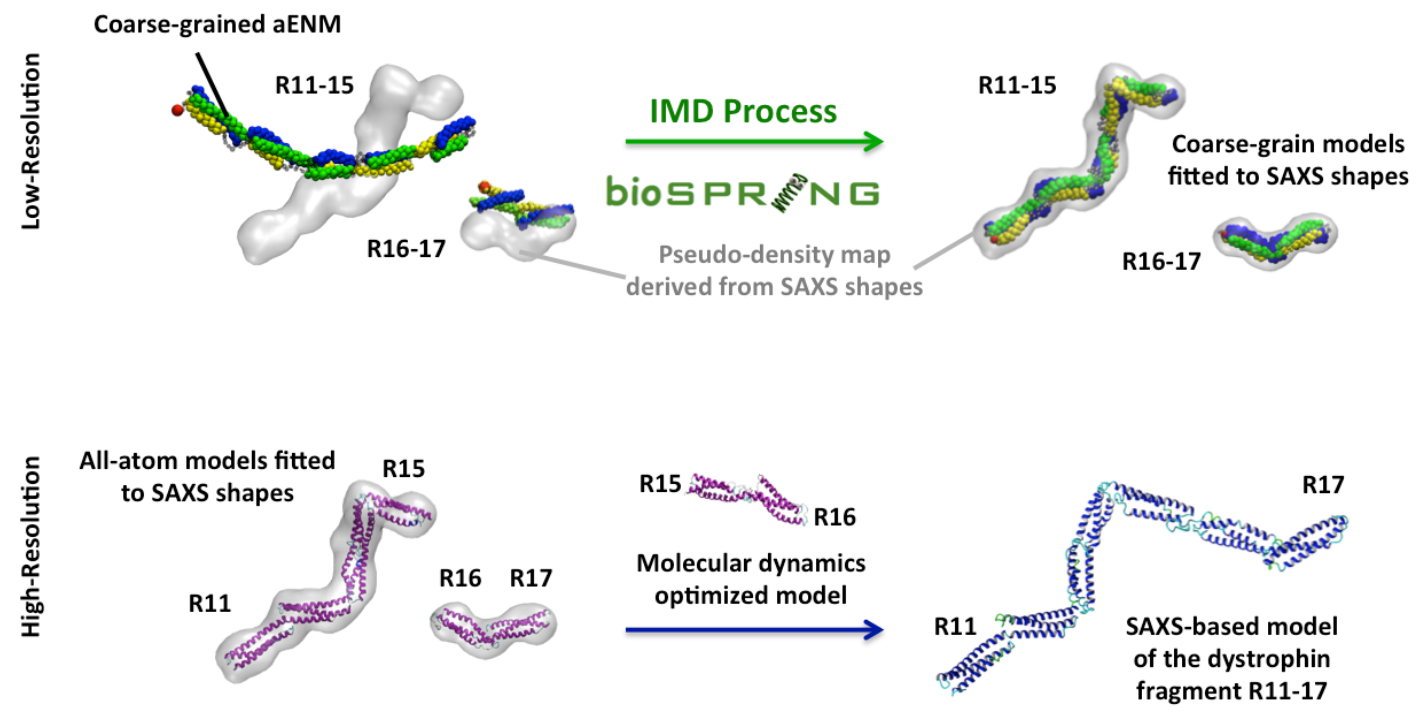

Figure 2B: (Top row) Schematic representation of BioSpring's interactive molecular dynamic processing of the R11-15 and R16-17 dystrophin fragments at lowresolution. For aENM, helices HA are yellow, helices HB are blue, and helices HC are green. The red spot represents the $\mathrm{N}$-terminal end; coils are gray. SAXS shapes were obtained from the GASBOR program of the ATSAS suite then converted to pseudo-density maps for driving the aENM model into the volumetric data. (Bottom row) Fragment reconstruction of an all-atom model is performed by superimposition onto the coarse-grain model then finalized by an optimization into the pseudo-density maps. The full-length filament is obtained by bridging the two SAXS-based models with an molecular dynamics-derived model of the R15-16 fragment, unavailable experimentally. 


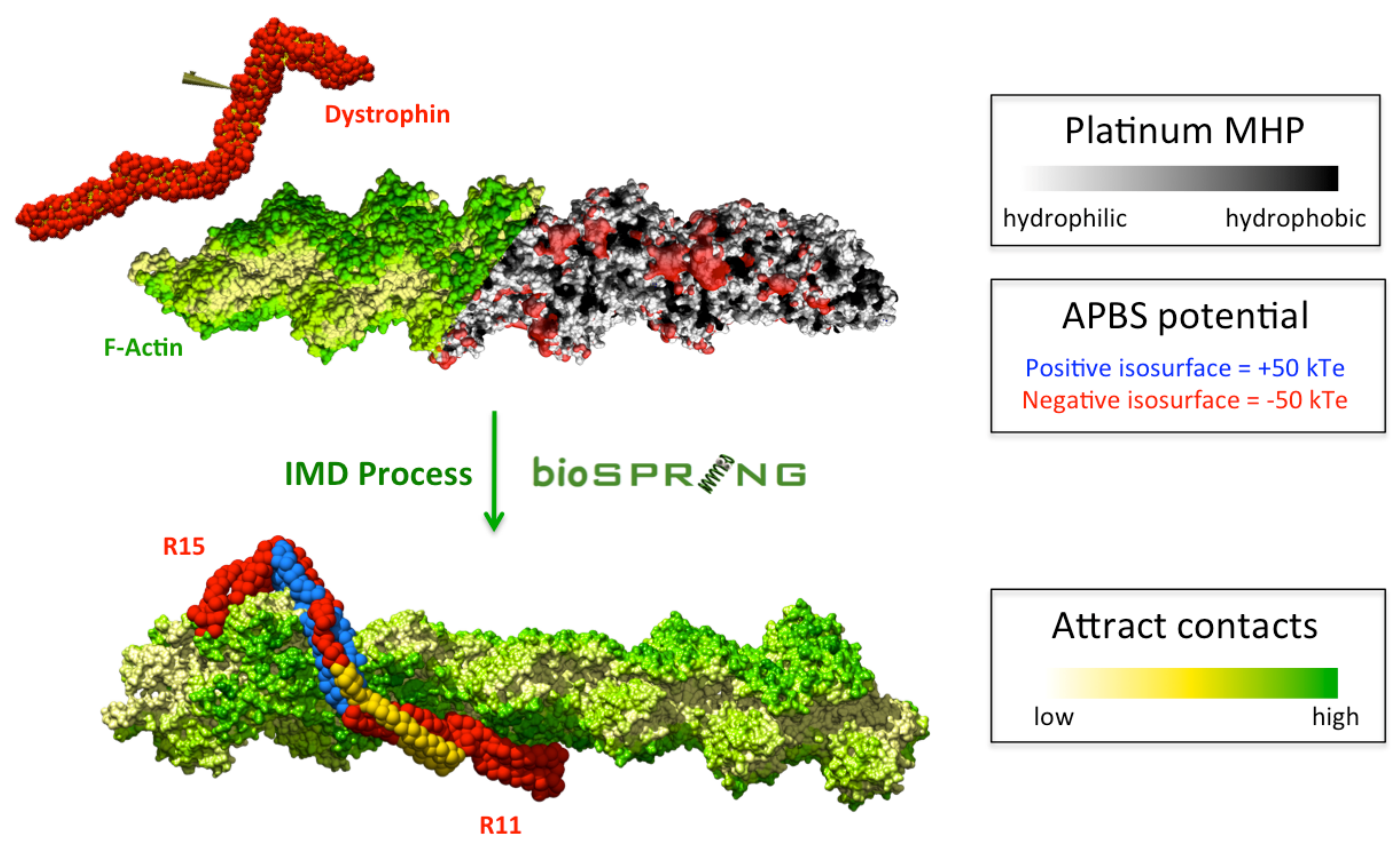

Figure 3: (Top) Starting point for Interactive Flexible Low-Resolution Docking. A dystrophin fragment (R11-15) is modelled by aENM shown here as red van der Waals spheres (coarse grain particles) and yellow sticks (springs). The actin filament's molecular surface (PDB code 3G37) is shown shading either from yellow to green by increasing probability of contact with dystrophin as indicated by Attract docking results, or from white (hydrophilic) to black (hydrophobic) according to Molecular Hydrophobicity Potential (MHP) provided Platinum server (32). The electrostatic potentials calculated using APBS software (27) are shown for $+/-50 \mathrm{kTe}$ isosurfaces and are blue (+) or red (-). These visual representations can be used to guide the user in the interactive experiments. (Bottom) Best pose of the R11-15 fragment after optimization of the aENM model into the potential grid computed for the actin filament structure. In the R11-15 dystrophin regions yellow residues are and blue are not protected against trypsin digestion upon the interaction of the protein with small unilamellar vesicles. 


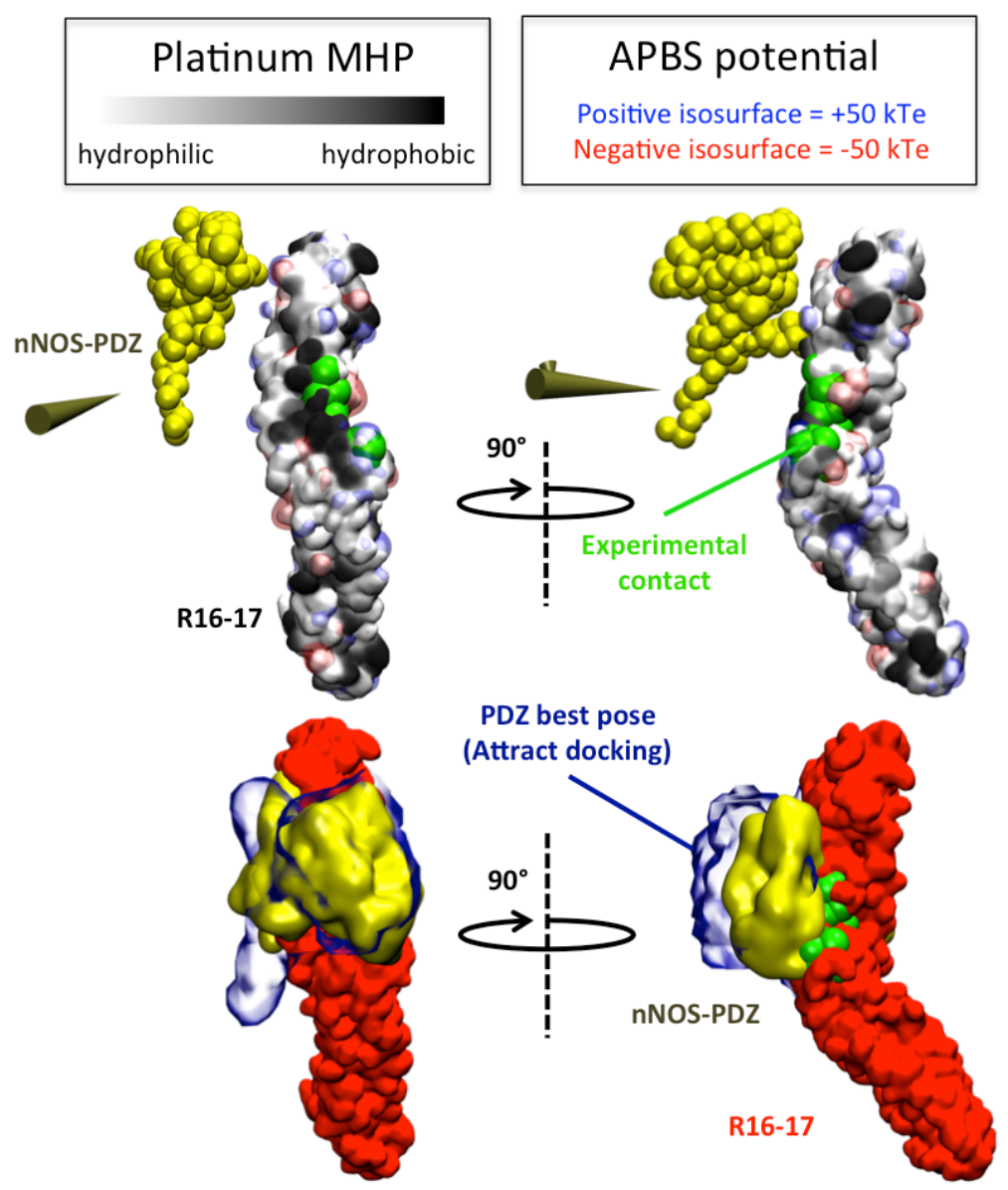

Figure 4: (Top) Starting point for Interactive Flexible Low-Resolution Docking. The dystrophin fragment (repeats R16-17) is shown with a molecular surface shading from white (hydrophilic) to black (hydrophobic) according to Platinum MHP (32). APBS potentials (27) are +/- $50 \mathrm{kTe}$ isosurfaces shown respectively in blue and red. The nNOS-PDZ subdomain is simulated by an aENM in yellow van der Waals spheres. The green spheres highlight the contact regions defined by mutagenesis performed in vitro and in vivo by Lai et al. (Bottom) The nNOS:Dystrophin complex obtained after interactive BioSpring docking, showing the best correlation with the experimental contact mapping. The blue phantom envelope corresponds to one of the best poses, based on complex energy and weighted after clustering, obtained from systematic rigid low-resolution docking (Attract). 
A

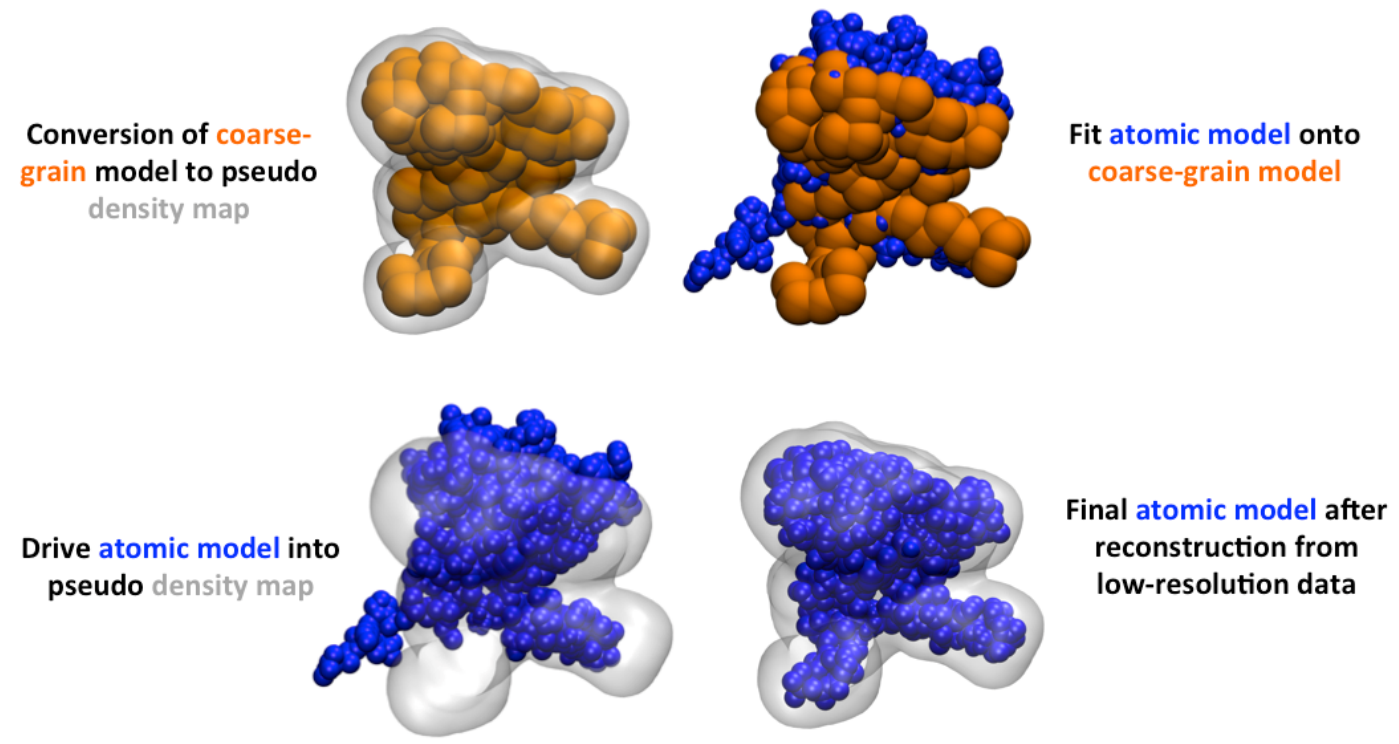

Figure 5A: BioSpring protocol for the atomic reconstruction of the low-resolution model obtained from interactive flexible docking.(Top left) The final position of the coarse-grain aENM in the molecular complex converted into a pseudo density map. (Top right) The atomic aENM is superimposed (C $\alpha$ RMSD) onto this same coarsegrain aENM final position. (Bottom left) The atomic aENM is driven into the pseudo density map. (Bottom right) The final atomic model is obtained after a few seconds of interactive BioSpring simulation. 
B
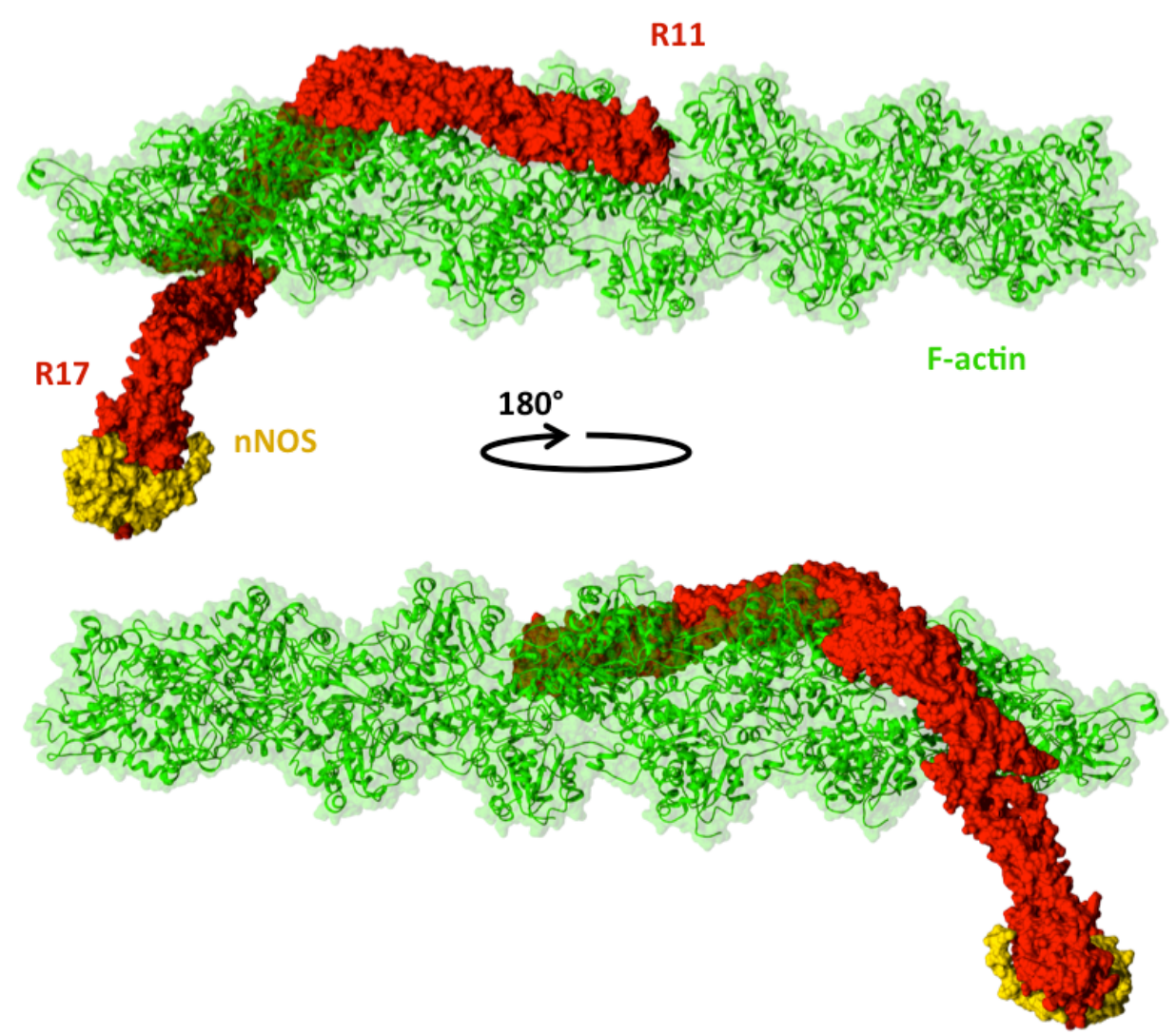

Figure 5B: Final ternary complex obtained by the association of dystrophin to F-actin and nNOS. Dystrophin is red, F-actin (PDB code 3G37) is green, and nNOS is yellow. The complex was developed by combining theoretical and experimental biostructural data with an interactive flexible approach. 
References

(1) Koenig, M., Monaco, A. P. and Kunkel, L. M. (1988) Cell 53, 219-226.

(2) Petrof, B. J., Shrager, J. B., Stedman, H. H., Kelly, A. M. and Sweeney, H. L. (1993) Proc Natl Acad Sci U S A 90, 3710-14.

(3) Koenig, M., Hoffman, E. P., Bertelson, C. J., Monaco, A. P., Feener, C. and Kunkel, L. M. (1987) Cell 50, 509-517.

(4) Le Rumeur, E., Hubert, J. F. and Winder, S. J. (2012) FEBS Letters 586, 271722.

(5) Le Rumeur, E., Winder, S. J. and Hubert, J. F. (2010) Biochim Biophys Acta 1804, 1713-1722.

(6) Winder, S. J., Gibson, T. J. and Kendrick-Jones, J. (1995) FEBS Letters 369, 2733.

(7) Amann, K.J., Renley, B.A. and Ervasti, J.M. (1998) A cluster of basic repeats in the dystrophin rod domain binds F-actin through an electrostatic interaction.

J. Biol. Chem. 273, 28419-28423.

(8) Rybakova, I.N., Amann, K.J. and Ervasti, J.M. (1996) A new model for the interaction of dystrophin with F-actin. J. Cell Biol. 135, 661-672.

(9) Lai, Y., Thomas, G. D., Yue, Y., Yang, H. T., Li, D., Long, C., Judge, L., Bostick, B., Chamberlain, J. S., Terjung, R. L. and Duan, D. (2009) J Clin Invest 119, 624635.

(10) Lai, Y., Zhao, J., Yue, Y. and Duan, D. (2012) Proc Natl Acad Sci U S A 110, 525-530.

(11) Legardinier, S., Raguenes-Nicol, C., Tascon, C., Rocher, C., Hardy, S., Hubert, J.-F. and Le Rumeur, E. (2009) J Mol Biol 389, 546-558.

(12) Sarkis, J., Vié, V., Winder, S. J., Renault, A., Le Rumeur, E. and Hubert, J. F. (2013) FASEB J 27, 359-67.

(13) Tuffery-Giraud, S., Béroud, C., Leturcq, F., Yaou, R. B., Hamroun, D., MichelCalemard, L., Moizard, M. P., Bernard, R., Cossée, M., Boisseau, P., Blayau, M., Creveaux, I., Guiochon-Mantel, A., de Martinville, B., Philippe, C., Monnier, N., Bieth, E., Khau Van Kien, P., Desmet, F. O., Humbertclaude, V., Kaplan, J. C., Chelly, J. and Claustres, M. (2009) Hum Mutat 30, 934-45.

(14) Gentil, C., Leturcq, F., Ben Yaou, R., Kaplan, J. C., Laforet, P., PenissonBesnier, I., Espil-Taris, C., Voit, T., Garcia, L. and Pietri-Rouxel, F. (2012) Hum Mol Genet 21, 3449-3460.

(15) Tek, A., Chavent, M., Baaden, M., Delalande, O., Bourdot, P. and Ferey, N. (2012) Advances in Human-Protein Interaction - Interactive and Immersive Molecular Simulations, Protein-Protein Interactions - Computational and Experimental Tools, Dr. Weibo Cai (Ed.), ISBN: 978-953-51-0397-4, InTech, DOI: 10.5772/36568.

(16) Saladin, A., Amourda, C., Poulain, P., Férey, N., Baaden, M., Zacharias, M., Delalande, O., \& Prévost, C. (2010). Nucleic Acids Research, 38, 6313-6323.

(17) Legrand, B., Giudice, E., Nicolas, A., Delalande, O. and Le Rumeur, E. (2011) Plos One 6, e23819.

(18) Roy, A., Kucukural, A. and Zhang Y. (2010) Nature Protocols, 5, 725-738.

(19) Muthu, M., Richardson, K. A. and Sutherland-Smith, A. J. (2012) PLoS One 7, e40066.

(20) Delarue, M. and Sanejouand, Y. H. (2002) J Mol Biol 320, 1011-24.

(21) Tama, F. and Sanejouand, Y. H. (2001) Protein Engineering 14, 1-6. 
(22) Schneider, S., Saladin, A., Fiorucci, S., Prévost, C. and Zacharias, M. (2012) Methods Mol Biol 819, 221-32.

(23) Zacharias, M. (2005) Proteins 60, 252-6.

(24) Periole, X., Cavalli, M., Marrink, S. J. and Ceruso, M. (2009) J Chem Theor Comp 5, 2531-43.

(25) Globisch, C., Krishnamani, V., Deserno, M. and Peter, C. (2013) PloS One 8, e60582.

(26) Case, D. A., Cheatham, T. E., III, Darden, T., Gohlke, H., Luo, R., Merz, K. M., Jr., Onufriev, A., Simmerling, C., Wang, B. and Woods, R. (2005) J Comput Chem 26, 1668-88.

(27) Baker, N. A., Sept, D., Joseph, S., Holst, M. J. and McCammon, J. A. (1998) Proc Natl Acad Sci U S A 98, 10037-10041.

(28) Petoukhov, M. V., Franke, D., Shkumatov, A. V., Tira, G., Kikhney, A. G., Gajda, M., Gorba, C., Mertens, H. D. T., Konarev, P. V. and Svergun, D. I. (2012) J Appl Cryst 45, 342-50.

(29) Humphrey, W., Dalke, A. and Schulten, K. (1996) J Mol Graph 14, 33-38

(30) Delalande, O., Férey, N., Grasseau, G. and Baaden, M. (2009) J Comput Chem 30, 2375-87.

(31) Pettersen, E. F., Goddard, T. D., Huang, C. C., Couch, G. S., Greenblatt, D. M., Meng, E. C. and Ferrin, T. E. (2004) J Comput Chem 25, 1605-12.

(32) Pyrkov, T. V., Chugunov, A. O., Krylov, N. A., Noldel, D. E. and Efremov, R. G. (2009) Bioinformatics 25, 1201-1202.

(33) Sarkis, J., Hubert, J. F., Legrand, B., Robert, E., Chéron, A., Jardin, J., Hitti, E., Le Rumeur, E. and Vié, V. (2011) J Biol Chem 286, 30481-91.

(34) Giudice, E., Molza, A. E., Laurin, Y., Nicolas, A., Le Rumeur, E. and Delalande, O. (2013) Biochemistry 52, 7777-84.

(35) Chan, K. Y., Gumbart, J., McGreevy, R., Watermeyer, J. M., Sewell B. T. and Schulten, K. (2011) Structure 19, 1211-18.

(36) Flores, S. C. (2013) Nucleic Acids Res, online, doi:10.1093/nar/gkt906 